\title{
Uterine metastasis of lobular breast carcinoma under tamoxifen therapy: A case report
}

\author{
YUICHIRO AWAZU, TAKESHI FUKUDA, KENJI IMAI, MAKOTO YAMAUCHI, MARI KASAI, \\ TOMOYUKI ICHIMURA, TOMOYO YASUI and TOSHIYUKI SUMI \\ Department of Obstetrics and Gynecology, Osaka City University Graduate \\ School of Medicine, Abeno-ku, Osaka 545-8585, Japan
}

Received September 28, 2021; Accepted October 14, 2021

DOI: $10.3892 / \operatorname{mco} .2021 .2428$

\begin{abstract}
Uterine metastases from breast cancer are uncommon and have rarely been reported in the previous literature. The present report describes the case of a 66-year-old female who developed uterine metastasis 23 years following the primary treatment of invasive breast cancer. Specifically, the patient experienced multiple bone metastases 14 years following primary treatment and had previously been treated with aromatase inhibitors followed by tamoxifen citrate. The patient presented with abnormal genital bleeding and was referred to the Gynecology Department of the Osaka City University Hospital (Osaka, Japan) 23 years following the primary treatment. The results of an endometrial biopsy revealed adenocarcinoma. Initially, it was difficult to differentiate between primary endometrial adenocarcinoma and metastatic adenocarcinoma from breast cancer. The results of pelvic magnetic resonance imaging demonstrated uterine myometrium enlargement and no endometrial thickness. Furthermore, an abdominal total hysterectomy, bilateral salpingo-oophorectomy and a biopsy of the peritoneum were performed. The pathological examination of the resected uterus revealed adenocarcinoma, which proliferated diffusively in the cervical stroma, myometrium, cardinal ligament, bilateral adnexa, omentum and peritoneum. Immunohistochemical results revealed the positive staining of gross cystic disease fluid protein-15, as well as negative staining for CD10 and E-cadherin. Thus, the tumor was diagnosed as metastatic adenocarcinoma from the breast lobular carcinoma. The patient has since been treated with fulvestrant, toremifene citrate and tegafur, and the current patient survival duration is 2 years and 8 months. In conclusion, when patients with breast cancer undergoing hormonal therapy, such as tamoxifen, present with abnormal genital bleeding, future diagnoses should consider both endometrial cancer and uterine metastasis from breast cancer.
\end{abstract}

Correspondence to: Dr Takeshi Fukuda, Department of Obstetrics and Gynecology, Osaka City University Graduate School of Medicine, 1-4-3 Asahimachi, Abeno-ku, Osaka 545-8585, Japan E-mail: takeshif@med.osaka-cu.ac.jp

Key words: uterine metastases, breast cancer, invasive lobular carcinoma, abnormal genital bleeding, tamoxifen

\section{Introduction}

Breast cancer frequently metastasizes to the liver, bone and lung (1) and only $8 \%$ of breast carcinomas are reported to metastasize to the uterus (2). Furthermore, only $3.8 \%$ of breast metastases to gynecological organs consist of metastases to the uterus (2). Metastasis of extragenital cancer to the female genital organs is uncommon and frequently involves metastasis to the ovaries (3). Metastases to the uterus from extragenital cancers are rare $[3.7 \%$ of metastatic tumors to the female genital tract from extragenital cancers $(2,4)]$ and are frequently caused by cancers of the gynecological organs, rather than extragenital sites (4). In addition, the most common type of primary cancer that causes uterine metastasis is breast cancer, accounting for $42.9 \%$ of uterine metastases, followed by colon cancer (17.5\%) and stomach cancer (11.1\%). In addition, the results of a previous study demonstrated that the uterine myometrium is more likely to be involved than the uterine endometrium (4). Metastasis to the myometrium remains asymptomatic; however, abnormal genital bleeding is a common symptom of metastasis when the endometrium has a role.

The most common type of breast carcinoma that metastasizes to the uterus is invasive lobular carcinoma (ILC) (5). After invasive ductal carcinoma (IDC), ILC is the second most common type of breast cancer, comprising of $10 \%$ of all invasive breast cancers (3). The results of a previous study demonstrated that ILC frequently metastasizes to distant lesions (3), and ILC frequently metastasizes to the peritoneum, ovaries and gastrointestinal tract (6).

The present report described the case of a 66-year-old female patient who developed uterine metastasis 23 years following the primary treatment of invasive breast cancer, who presented with abnormal genital bleeding.

\section{Case report}

A 66-year-old female (gravida 5, para 3) was referred to the Gynecology Department of Osaka City University Hospital (Osaka, Japan) due to abnormal genital bleeding lasting for 2 months. The patient experienced menopause at the age of 56 years. The patient was also diagnosed with right invasive lobular breast cancer at the age of 43 years and 

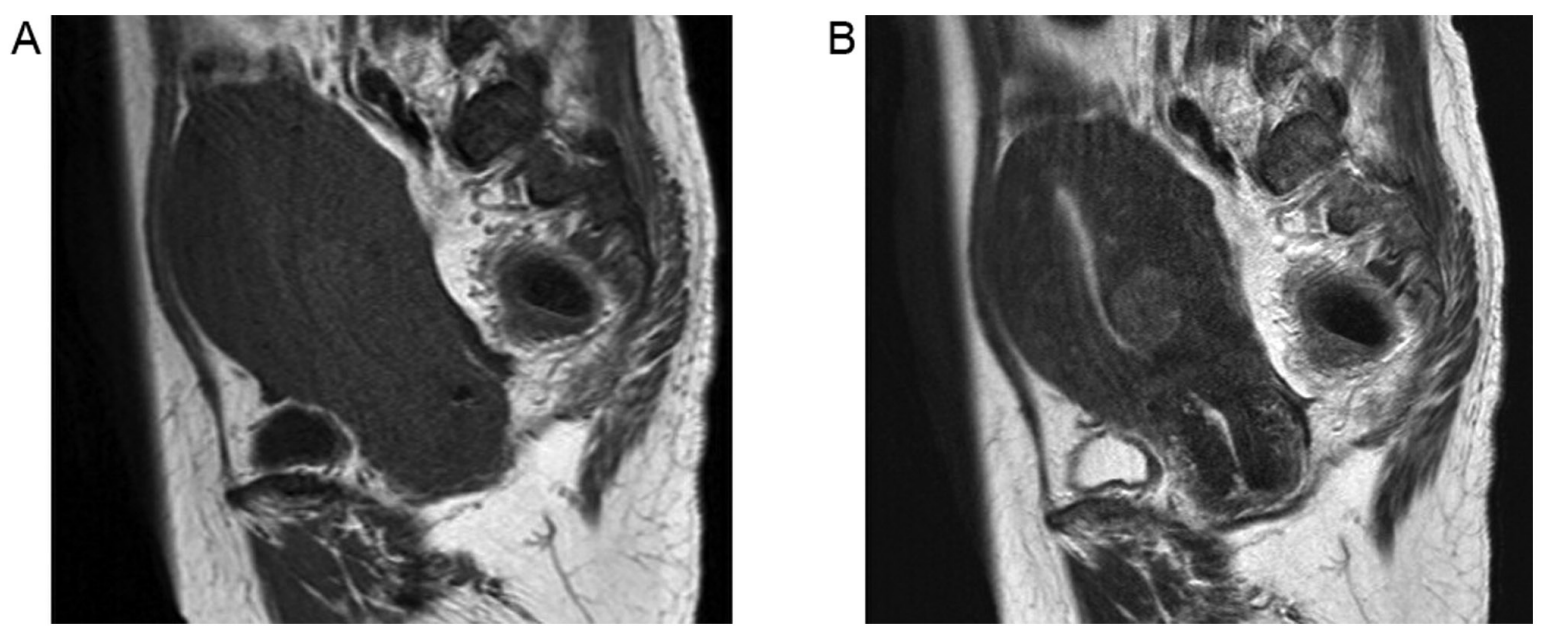

Figure 1. Magnetic resonance imaging of the pelvis. (A) T1-weighted sagittal image. (B) T2-weighted sagittal image. Results revealed that the thickness of the endometrium was $7 \mathrm{~mm}$ and no notable mass was present in the endometrium.
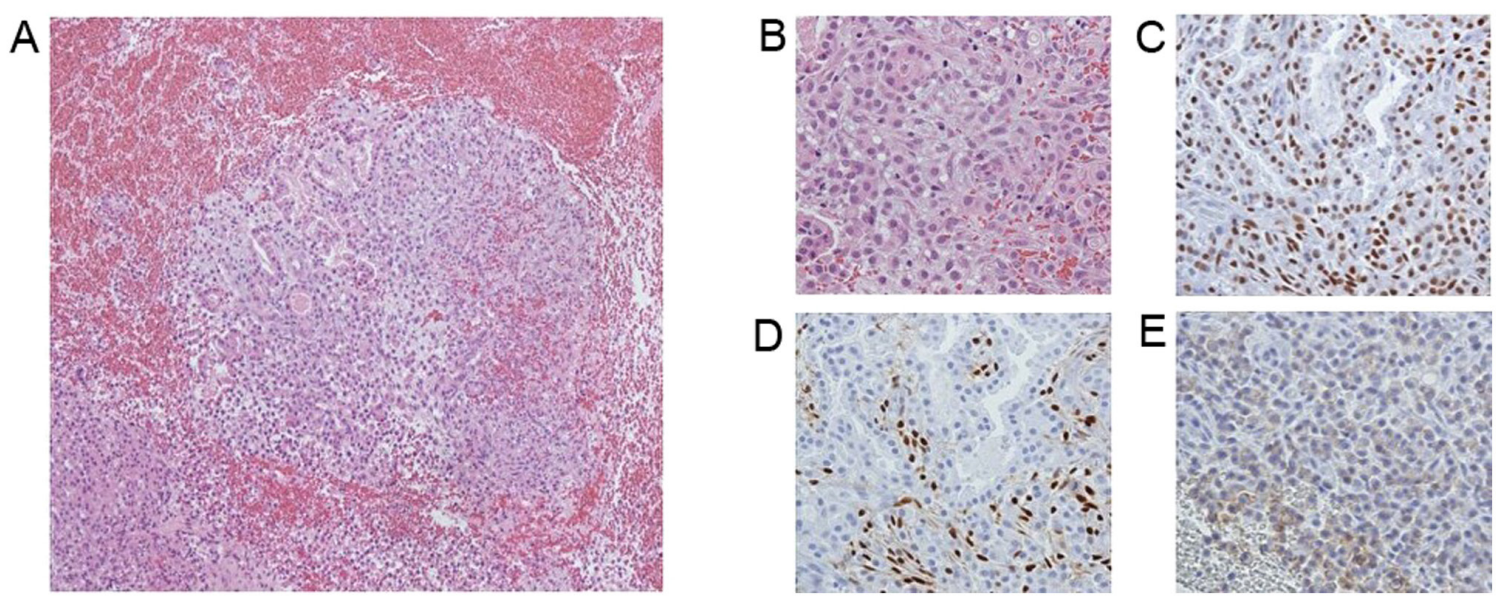

Figure 2. Pathological examination of the endometrium biopsy. (A) H\&E staining (magnification, x100). (B) H\&E staining revealed cancer cells with rich cytoplasm and the proliferation of lumen-forming cells (magnification, x200). Immunohistochemical analysis of (C) estrogen receptor, (D) progesterone receptor and (E) human epidermal growth factor receptor 2 (magnification, x200).

received right breast resection and chemotherapy (detailed treatment is unknown due to a lack of medical records). The patient was further diagnosed with recurrence on the right breast and multiple bone metastases at the age of 60 years and was subsequently treated with aromatase inhibitors followed by tamoxifen citrate. At 23 years after the primary surgery (age, 66 years), the patient presented with abnormal genital bleeding lasting for 2 months. Gynecological examination revealed a small amount of bloody vaginal discharge and an enlarged uterine cervix. The uterine corpus was also enlarged and mobility was restricted. It was not possible to palpitate the bilateral adnexa. Magnetic resonance imaging of the pelvis revealed that the thickness of the endometrium was $7 \mathrm{~mm}$ and there was no notable mass in the endometrium (Fig. 1). The myometrium of the uterus was enlarged, which appeared to be due to the presence of adenomyosis. Furthermore, a 15-mm degenerated myoma was present on the posterior myometrium. Computed tomography scan of the chest and abdomen revealed multiple bone metastases, which had previously been reported, and no evidence of metastasis to the other organs. Uterine cervical cytology revealed no malignancy. Biopsy of the endometrium and routine staining for estrogen receptor $(\mathrm{ER} ;++)$, progesterone receptor $(\mathrm{PR} ;+/$-) and human epidermal growth factor receptor 2 (HER2; +/-) revealed adenocarcinoma. It was therefore difficult to differentiate whether this was metastasis from breast cancer or primary endometrial adenocarcinoma (Fig. 2). Abdominal total hysterectomy, bilateral salpingo-oophorectomy, partial omentectomy and biopsy of the peritoneum were subsequently performed. Multiple millet-sized metastases were observed at the peritoneum, mesentery, surface of the intestines, omentum and diaphragm. On gross inspection following specimen removal, diffuse enlargement of the myometrium and irregular surface of the endometrium were revealed (Fig. 3). As displayed in Fig. 4, the results of the pathological examination revealed diffuse invasion of breast lobular cancer to the cervical stroma, myometrium, cardinal ligament, bilateral adnexa, omentum and peritoneum. Immunohistochemical analysis demonstrated ER (++), PR (+/-), HER2 (+/-), CD10 (-), CAM5.2 (+), gross cystic disease fluid protein-15 (GCDFP; + ) and E-cadherin (-). Negative staining for CD10 indicated that the tumor had not originated from the endometrium. Positive staining for 


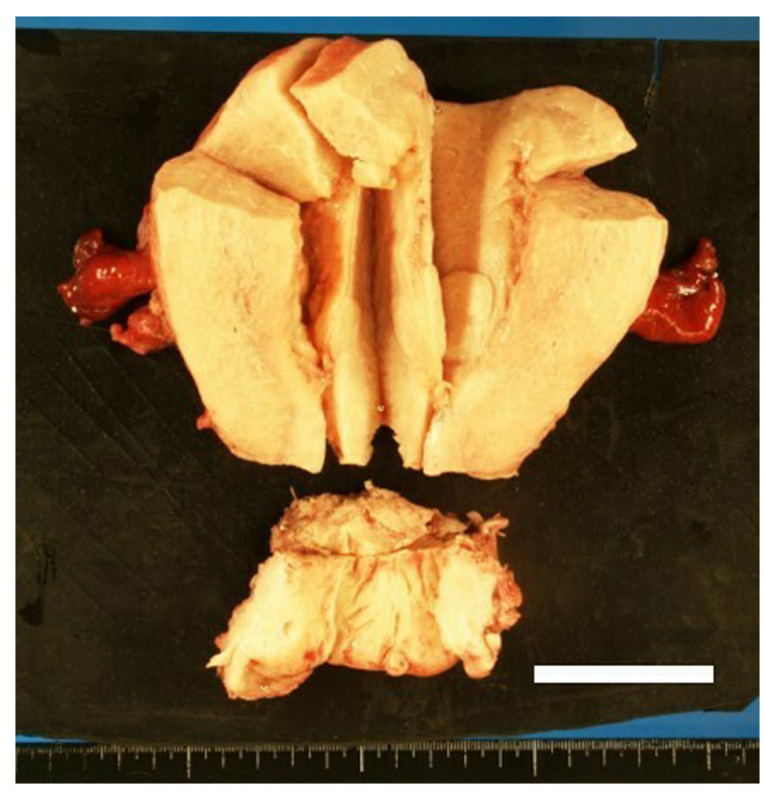

Figure 3. Macroscopic image of resected uterus and bilateral adnexa. Diffuse enlargement of the myometrium and irregular surface of the endometrium were observed (scale bar, $5 \mathrm{~cm}$ ).
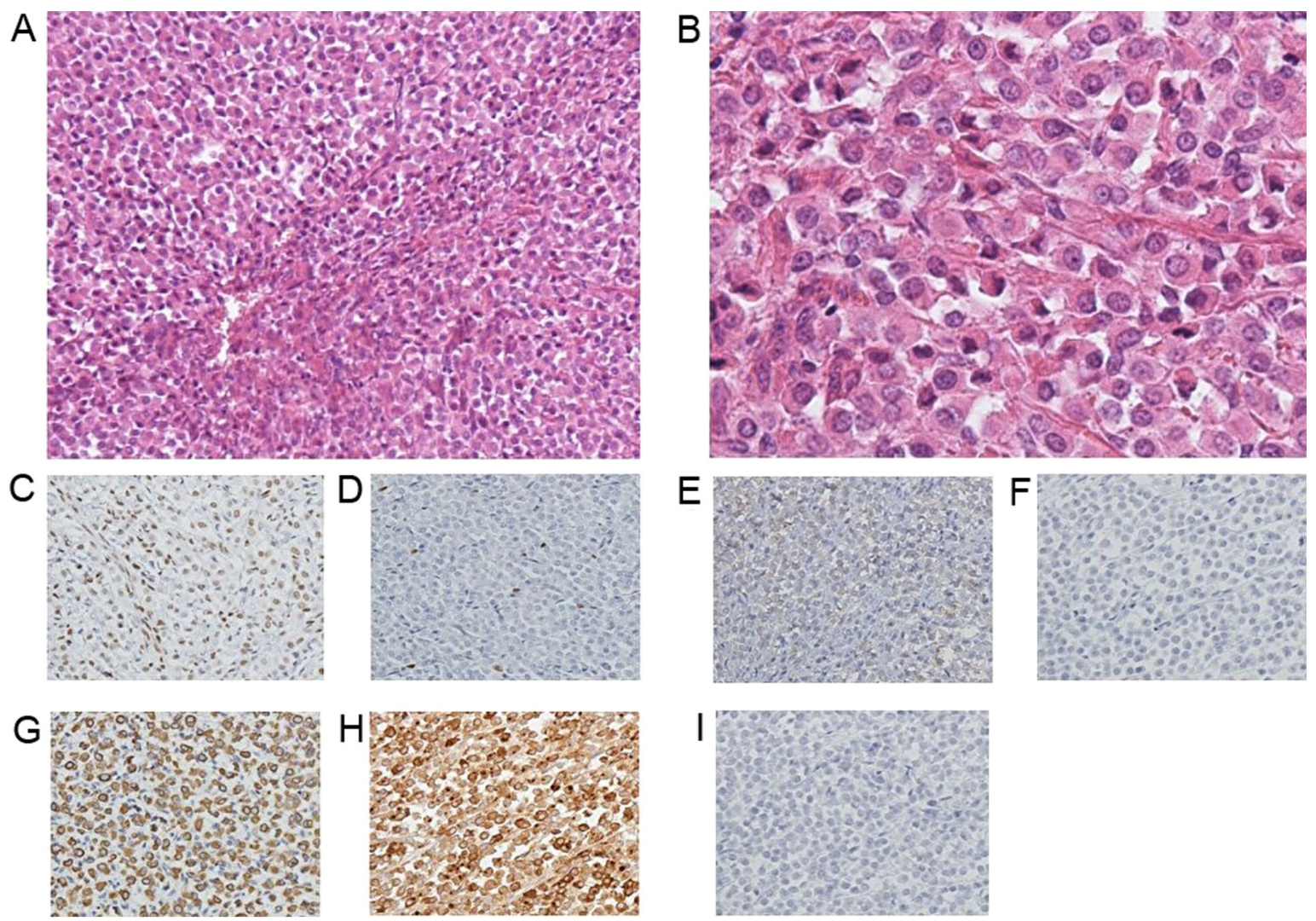

Figure 4. Pathological examination of the resected uterus. (A) H\&E staining (magnification, x100). (B) H\&E staining revealed cancer cells with oval nuclei, reduced cytoplasm and the formation of solid nests (magnification, x400). Immunohistochemical analysis of (C) estrogen receptor, (D) progesterone receptor, (E) human epidermal growth factor receptor 2, (F) CD10, (G) CAM5.2, (H) gross cystic disease fluid protein-15 and (I) E-cadherin (magnification, x200).

GCDFP indicated that the tumor had originated from the breast and negative staining for E-cadherin suggested that the tumor was lobular carcinoma, rather than ductal carcinoma. The patient has since been treated with fulvestrant, toremifene citrate and tegafur, and the current patient survival duration is 2 years and 8 months.

\section{Discussion}

Mazur et al (2) reported that the most frequently affected female genital tract by metastatic disease from extragenital cancers is the ovaries (75\%), followed by the vagina (5.8\%), endometrium (3.8\%), cervix (3.4\%) and vulva (2\%) (2). Of 
note, the breast and gastrointestinal tract are the most common origins of primary tumors that subsequently metastasize to the female genital tract (4). When uterine metastasis is diagnosed, the most frequent origin of the primary tumor is breast cancer (42.9\%) (4). Furthermore, results of a previous study demonstrated that $63.5 \%$ of uterine corpus metastasis cases involved the myometrium alone, $32.7 \%$ of the cases involved both the myometrium and endometrium and $3.8 \%$ of the cases involved the endometrium alone (2).

The ovaries are frequently involved in the spread of metastatic cancer cells (2). The rich vasculature and well-developed lymph network of the ovaries provide a favorable environment for the implantation of cancer cells. Of note, both the $\mathrm{pH}$ levels and oxygen pressure in the stroma of the ovaries are favorable for the growth and development of cancer cells $(7,8)$. Retrograde lymphatic spreading of an ovary-originating primary tumor causes metastasis to the uterus in $\sim 60 \%$ of patients; however, cases of metastases to the uterus without affected ovaries are rare and may be caused by hematogenous spread (4).

Breast cancer is the most common malignancy in females. A total of $>2$ million breast cancer cases are diagnosed per year and breast cancer leads to $>600,000$ cancer-associated mortalities worldwide (9). Due to advances in breast cancer treatments and early diagnoses, the breast cancer survival rate is improving (10). Consequently, the number of females living with metastatic breast cancer has increased (11).

The two most frequent histological subtypes of breast cancer are IDC and ILC (3). A total of $\sim 76 \%$ of all breast cancers are categorized as IDC, and $\sim 8 \%$ of all breast cancers are categorized as ILC (3). Although the incidence rate remains low, ILC frequently metastasizes to the female genital tract (4). Frequent metastasis of ILC is caused by reduced levels of expression of the cellular adhesion molecule E-cadherin, which is not observed in IDC (12). ILC is also characterized by reduced sensitivity to neoadjuvant chemotherapy compared with IDC (13).

Tamoxifen is a widely-established oral drug for the treatment of ER-positive breast cancer. Tamoxifen acts as a selective ER modulator in the breast; however, it also behaves as an estrogen agonist on the endometrium, resulting in increased levels of carcinogenesis (14). Thus, patients with breast cancer who are treated with tamoxifen may suffer from uterine metastases, as well as primary endometrial cancer.

Abnormal genital bleeding is the most common initial manifestation of primary endometrial cancer and is also the first symptom of endometrial metastases (4). Abnormal genital bleeding occurs when the endometrium or the cervix is involved in tumor development and progression. Thus, patients with a previous history of breast cancer and treatment with tamoxifen who present with abnormal genital bleeding must be examined for primary endometrial cancer and metastatic uterine cancer.

Both the clinical history of the patient and pathological examination, including immunohistochemical analysis, are required in order to differentiate between metastatic breast cancer and primary endometrial cancer, as their treatment strategy and prognosis exhibit marked differences. A key biomarker for the differentiation of primary endometrial cancer from metastatic breast cancer is GCDFP. GCDFP is a glycoprotein originally detected in the cystic fluid following cystic mastopathy and is therefore a useful immunohistochemical marker to evaluate the potential mammary origin of a tumor when the primary site remains to be elucidated (15). In the present case report, metastatic uterine cancer was successfully diagnosed following positive immunohistochemical staining for GCDFP.

In conclusion, the present report described the case of a 66-year-old female who developed uterine metastasis 23 years after the primary treatment of invasive lobular breast cancer. The patient also presented with abnormal genital bleeding. Thus, the present report highlights the importance of considering not only primary endometrial cancer, but also uterine metastasis from breast cancer in the diagnostic process. This consideration is crucial when a patient with breast cancer undergoing hormonal therapy, such as tamoxifen, presents with abnormal genital bleeding, despite the rarity of uterine metastases from breast cancer.

\section{Acknowledgements}

Not applicable.

\section{Funding}

This study was funded by The Osaka Medical Research Foundation for Intractable Diseases (grant no. 27-2-4).

\section{Availability of data and materials}

The datasets used and/or analyzed during the current study are available from the corresponding author upon reasonable request.

\section{Authors' contributions}

YA, TF and TS conceived and designed the study. YA, TF, KI, MY, MK, TI and TY acquired, analyzed and interpreted the data. YA, TF and TS drafted and revised the manuscript. TF and TS confirm the authenticity of all the raw data. All authors have read and approved the final manuscript.

\section{Ethics approval and consent to participate}

Not applicable.

\section{Patient consent for publication}

Written informed consent was obtained from the patient for the publication of the case details and any associated images.

\section{Competing interests}

The authors declare that they have no competing interests.

\section{References}

1. Almagro E, González CS and Espinosa E: Prognostic factors of early breast cancer. Med Clin (Barc) 146: 167-171, 2016 (In Spanish).

2. Mazur MT, Hsueh S and Gersell DJ: Metastases to the female genital tract. Analysis of 325 cases. Cancer 53: 1978-1984, 1984. 3. Watkins EJ: Overview of breast cancer. JAAPA 32: 13-17, 2019. 
4. Kumar NB and Hart WR: Metastases to the uterine corpus from extragenital cancers. A clinicopathologic study of 63 cases Cancer 50: 2163-2169, 1982.

5. Harris M, Howell A, Chrissohou M, Swindell RI, Hudson M and Sellwood RA: A comparison of the metastatic pattern of infiltrating lobular carcinoma and infiltrating duct carcinoma of the breast. Br J Cancer 50: 23-30, 1984.

6. Arpino G, Bardou VJ, Clark GM and Elledge RM: Infiltrating lobular carcinoma of the breast: Tumor characteristics and clinical outcome. Breast Cancer Res 6: R149-R156, 2004.

7. Perisić D, Jancić S, Kalinović D and Cekerevac M: Metastasis of lobular breast carcinoma to the cervix. J Obstet Gynaecol Res 33: 578-580, 2007.

8. Razia S, Nakayama K, Tsukao M, Nakamura K, Ishikawa M, Ishibashi T, Ishikawa N, Sanuki K, Yamashita H, Ono R, et al: Metastasis of breast cancer to an endometrial polyp, the cervix and a leiomyoma: A case report and review of the literature. Oncol Lett 14: 4585-4592, 2017.

9. Bray F, Ferlay J, Soerjomataram I, Siegel RL, Torre LA and Jemal A: Global cancer statistics 2018: GLOBOCAN estimates of incidence and mortality worldwide for 36 cancers in 185 countries. CA Cancer J Clin 68: 394-424, 2018.

10. Giordano SH, Buzdar AU, Smith TL, Kau SW, Yang Y and Hortobagyi GN: Is breast cancer survival improving? Cancer 100: 44-52, 2004
11. Mariotto AB, Etzioni R, Hurlbert M, Penberthy L and Mayer M: Estimation of the number of women living with metastatic breast cancer in the United States. Cancer Epidemiol Biomarkers Prev 26: 809-815, 2017.

12. Li CI, Uribe DJ and Daling JR: Clinical characteristics of different histologic types of breast cancer. Br J Cancer 93: 1046-1052, 2005.

13. Fu D, Zuo Q, Huang Q, Su L, Ring HZ and Ring BZ: Molecular Classification of Lobular Carcinoma of the Breast. Sci Rep 7: 43265, 2017.

14. Barakat RR: Tamoxifen and endometrial neoplasia. Clin Obstet Gynecol 39: 629-640, 1996.

15. Darb-Esfahani S, von Minckwitz G, Denkert C, Ataseven B, Högel B, Mehta K, Kaltenecker G, Rüdiger T, Pfitzner B, Kittel K, et al: Gross cystic disease fluid protein 15 (GCDFP-15) expression in breast cancer subtypes. BMC Cancer 14: 546, 2014.

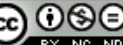

This work is licensed under a Creative Commons Attribution-NonCommercial-NoDerivatives 4.0 International (CC BY-NC-ND 4.0) License. 